\title{
Plantar Fascial Rupture of the Foot: A case report
}

\author{
by Al Kline, $\mathrm{DPM}^{1} \square$
}

The Foot and Ankle Online Journal 2 (5): 4

Plantar fascial rupture is rarely presented in the literature. Spontaneous rupture of the plantar fascia is commonly preceded by plantar fasciitis. A 60 year old male presents following an acute injury of his foot while playing softball. He presents with acute pain and ecchymosis to the plantar arch of the foot. Plantar fascial rupture was diagnosed clinically and confirmed on magnetic resonance imaging (MRI). This case discusses the clinical evaluation, MRI results and treatment of acute, spontaneous rupture of the plantar fascia. We also describe the MRI differences of plantar fasciitis and plantar fascial rupture.

Key words: Plantar fasciitis, plantar fascial rupture, heel pain, Magnetic resonance imaging

Accepted: April, 2009

Published: May, 2009

This is an Open Access article distributed under the terms of the Creative Commons Attribution License. It permits unrestricted use, distribution, and reproduction in any medium, provided the original work is properly cited. (The Foot and Ankle Online Journal (www.faoj.org)

$\mathrm{P}$ lantar fascial injuries are a common source of foot pain. Plantar fasciitis is the most common type of plantar fascial injury. The condition is characterized by small tears of the plantar aponeurosis that can cause inflammation and thickening of the plantar aponeurosis. The causes of injury are related most commonly to stress and strain. General injury to the plantar fascia can be divided into three categories: mechanical, degenerative and systemic. ${ }^{1}$ Mechanical conditions such as pronation, forefoot varus and rearfoot valgus will often lead to increased tension and strain of the plantar aponeurosis. This may be exacerabated by increased activity and lack of proper shoe and in-step support. It is now widely accepted that degenerative changes can occur within the plantar fascia due to repetitive micro tears and perifascial edema termed plantar fasciosis. ${ }^{2}$ This is characterized as a degenerative process of myxoid degeneration without inflammation. ${ }^{2}$

Address correspondence to: Al Kline, DPM

3130 South Alameda, Corpus Christi, Texas 78404.

\footnotetext{
${ }^{1}$ Adjunct Clinical Faculty, Barry University School of Podiatric Medicine. Private practice, Chief of Podiatry, Doctors Regional Medical Center. Corpus Christi, Texas, 78411.
}

There are also a number of inflammatory systemic conditions that can cause plantar fasciitis. These include rheumatoid arthritis, ankylosing spondylitis, psoriatic arthritis, Reiter's syndrome, gout, Behcet's Syndrome and systemic lupus erythematosus. ${ }^{3}$ In general, the etiology of arch and heel pain can be mulifactorial in nature. When tension along the plantar aponeurosis exceeds its inherent strength, an acute fascial rupture can result.

\section{Case Report}

A 60-year old healthy male presented to our office in acute pain. He presented with a limp. He stated that he had been having arch and heel pain of the right foot over the past month. He recently participated in a softball game. He states that while 'sprinting' to a base, he felt a 'pop' in his arch followed by acute pain and swelling. He immediately stopped playing and placed ice on the arch region of the foot. Clinical evaluation of the foot reveals an extremely tender plantar fascia with localized bruising or ecchymosis (Fig.1). 


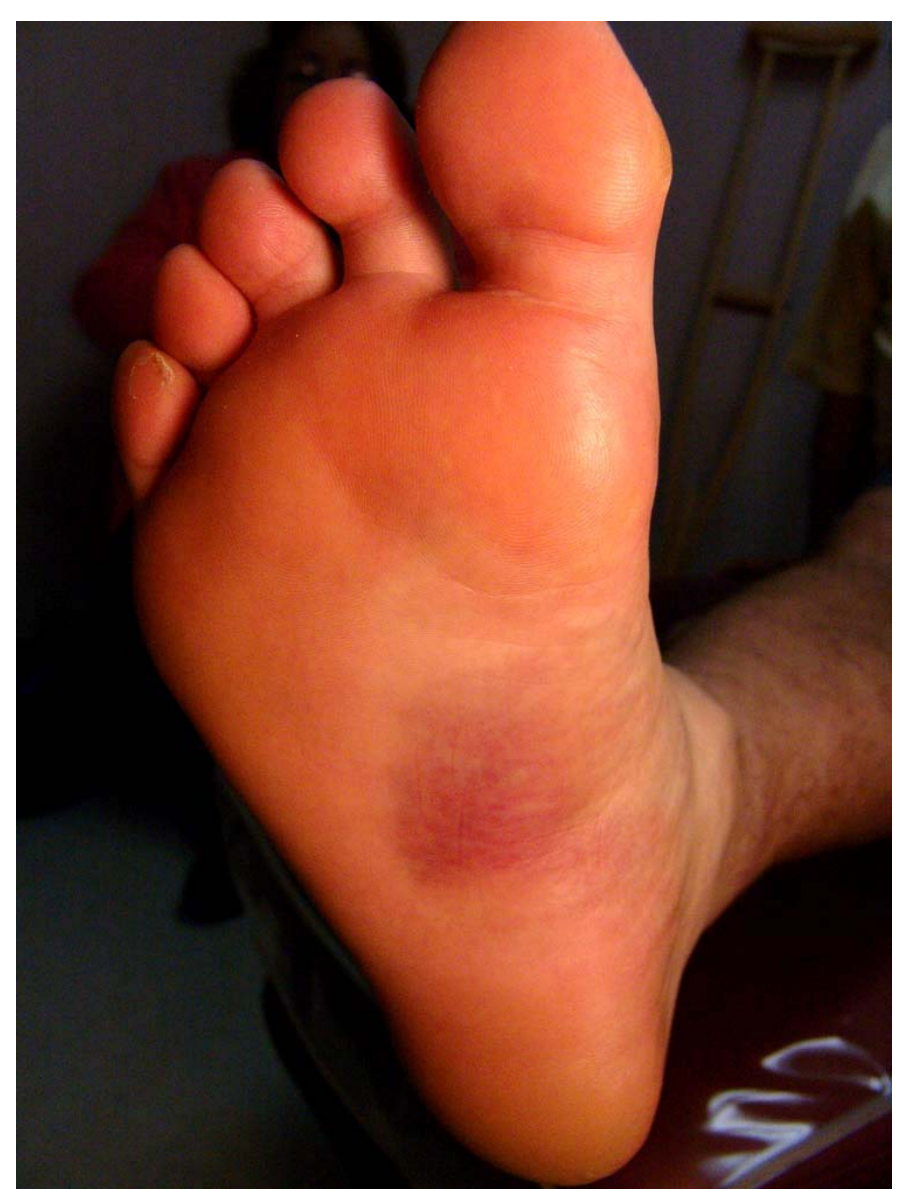

Figure 1 The plantar fascia shows bruising directly along the arch of the foot. There is extreme point tenderness to this region.

Pain was palpable along the entire course of the plantar fascia and more pronounced along the central arch. The patient was sent for magnetic resonance imaging (MRI) confirmation to rule out plantar fascial rupture. Pain was palpable along the entire course of the plantar fascia and more pronounced along the central arch. The patient was sent for MRI confirmation to rule out plantar fascial rupture.

\section{MR Imaging and Findings}

MRI shows classic signs of fascial tear and rupture. Multiplanar, multisequence images were obtained showing increased thickness of the plantar fascia up to $10 \mathrm{~mm}$ with convexed dorsal thickening. A classic fusiform appearance of the fascia is seen in the region of rupture.

(C) The Foot and Ankle Online Journal, 2009

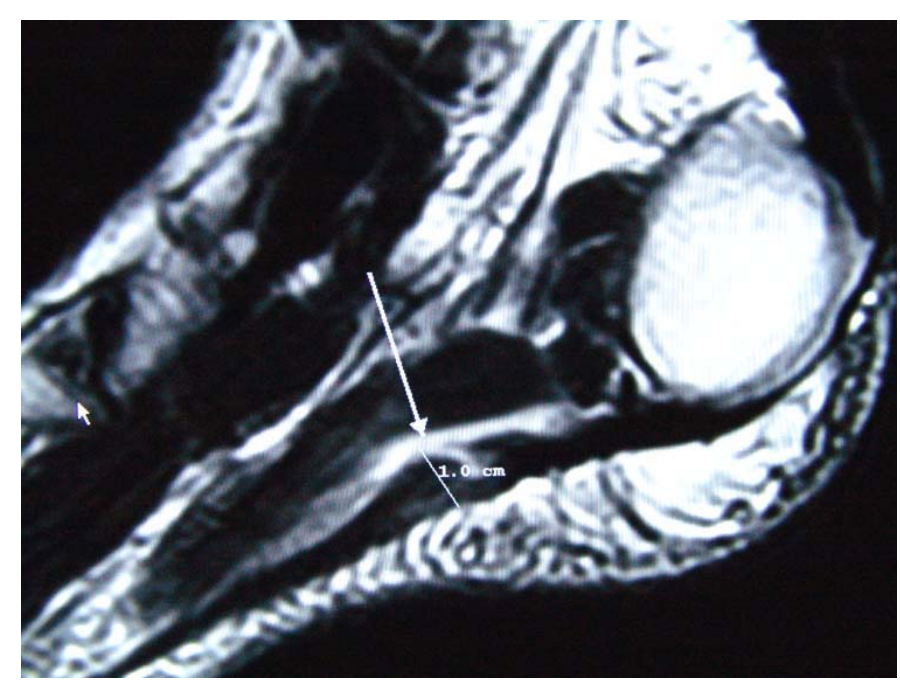

Figure 2 T2 sagittal image shows a central thickening up to $10 \mathrm{~mm}$ with enlargement and nodular thickening of the plantar aponeurosis.

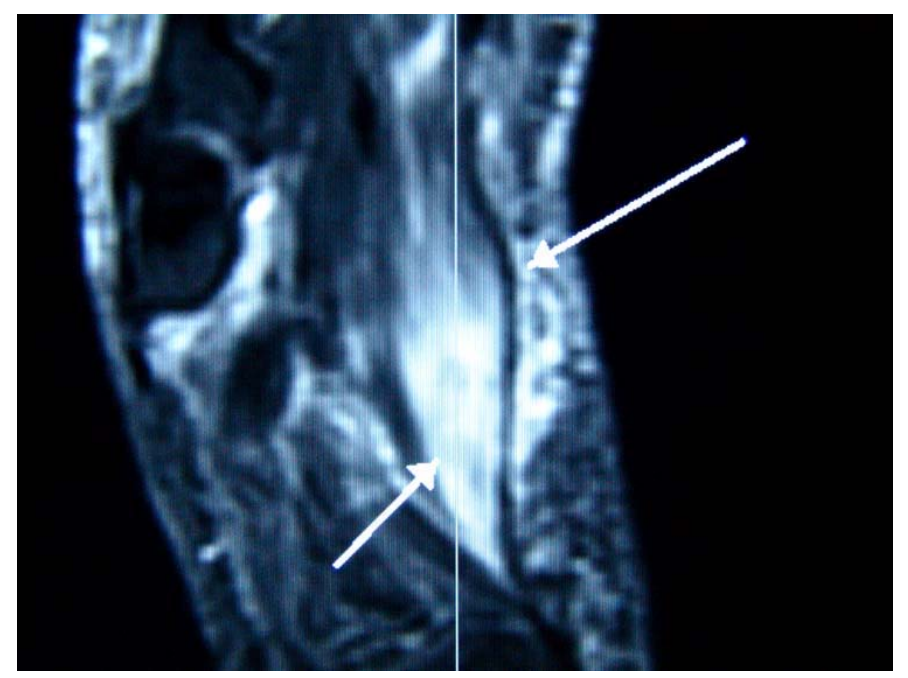

Figure 3 STIR (inversion recovery image) coronal views also shows intrafascial edema and hemorrhage.

The sagittal image also shows intrafascial high signal echo on T2 imaging consistent with plantar fascial disruption of the fibers. (Fig. 2)

Perifascial edema (arrow) is seen along the deeper musculature adjacent to the plantar aponeurosis. The coronal view on STIR or inversion recovery sequencing shows dramatic intrafascial edema and hemorrhage. Again, fusiform thickening of the musculature and plantar aponeurosis is appreciated. (Fig. 3) 


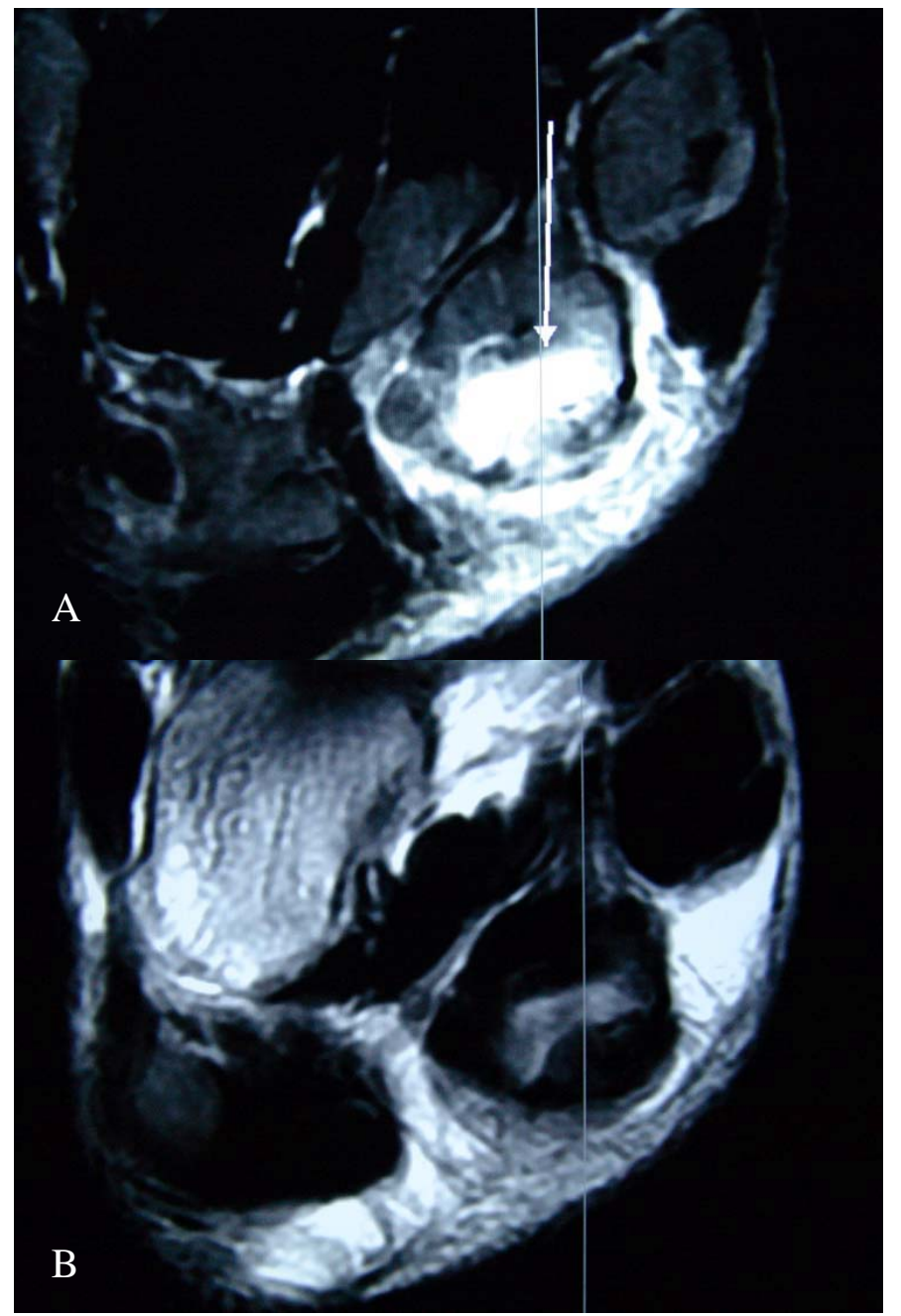

Figure 4A and 4B MR Axial imaging shows T1 image (A). The T2 image shows increased perifascial, intrafascial and muscular edema. (B)

Axial imaging shows increased signal intensity on T1 and T2 imaging with appreciable intrafascial and perifascial edema. (Fig. $4 \mathrm{~A}$ and B)

\section{Treatment}

Treatment of plantar fascia rupture depends on the extent of injury confirmed by MRI findings and activity level of the patient. Our patient was active for his age and his overall injury was acute and extremely painful. In this respect, we recommended the patient wear a non-weight bearing cast for 4 weeks.
We placed him on NSAIDS for 2 weeks during his casting period. His recovery after casting included local stretching and physical therapy. We also placed him in orthotics.

\section{Discussion}

The clinical presentation of acute plantar fascial rupture differs from plantar fasciitis. The pain of an acute rupture is located more distal to the insertion of the plantar fascia and bruising is commonly seen along the middle of the arch. Clinically, this is extremely tender to touch and the patient will have trouble walking. Most often, clinical evaluation, activity of the patient and onset of pain will help the practitioner determine the extent of injury and determine fascial strain or fasciitis from actual tear or rupture of the plantar fascia. Radiographic evaluation lacks the proper contrast resolution for proper differentiation of plantar fasciitis and fascial rupture. Fascial thickening and perifascial edema can be seen on enhanced soft tissue radiographic imaging. However, MR imaging is superior in differentiating acute plantar fasciitis, chronic plantar fasciitis from partial or acute plantar fascial rupture. MR imaging will determine the exact localization and extent of fascial injury. In this regard, the proportionate thickness and amount of edema will help the practitioner determine the proper course of treatment.

The attachment of the plantar fascia is best demonstrated on coronal images. The entire course of the aponeurosis is best seen on the sagittal images. Visualizing of the medial fascial band is best seen in the sagittal and coronal views. The lateral band is best observed with oblique imaging, although sagittal and coronal images can also be used. MR imaging studies also show a difference in findings when comparing fasciitis and fascial rupture. In plantar fasciitis, there is often thickening of the aponeurosis as seen on sagittal image without actual disruption of the fascial fibers. The appearance of the plantar fascia is usually thickened and uniform. In plantar fascial rupture, there is often a fusiform appearance of the aponeurosis. 
There is also widespread abnormal high signal intensity infiltrating perifascial soft tissues consistent with local edema. The most consistent finding in acute partial or complete rupture of the plantar aponeurosis is fusiform thickening of the fascia with abnormal, intrafascial signal intensity. Theodorou, et al., studied MR imaging of 14 patients with partial or complete rupture of the plantar fascia revealing abnormal, fusiform thickening of the plantar aponeurosis in all patients. All patients showed abnormal absence of T1-weighted low signal intensity of the plantar aponeurosis at the site of complete rupture or partial loss of T1-weighted low signal intensity respectively. ${ }^{1}$

Treatment can vary on extent of injury and activity of the patient. In earlier studies and before MR imaging techniques, patients with rupture were often treated conservatively using crutches, ice packs, antiinflammatory agents and foot straps. Diagnosis was simply made by presentation of acute symptoms such as severe localized swelling and acute tenderness. As the swelling diminished, there is often a palpable defect that is replaced by a hard mass that gradually became less tender. ${ }^{4}$ Leach, et al., reported suspected partial ruptures in six long distance runners who were treated conservatively. Only one patient required surgery for persistent swelling, undergoing a fascial release. They reported full recovery of all the long distance runners back to their original pre-injury activity with no deleterious effects, even in the one surgical patient. Now, with the aid of MR imaging, diagnosis and treatment can be more specific to extent of injury.

This case highlights clinical and MRI findings in a patient with plantar fascial rupture following an acute injury while playing softball. A fusiform appearance of the fascia on MR imaging was consistent with plantar fascial rupture and the patient's clinical presentation. In its largest point, the intrafascial edema can increase the thickness of the fascia to over $10 \mathrm{~mm}$. The normal thickness of the plantar fascia is about $4 \mathrm{~mm}$ in thickness. In cases of plantar fasciitis, the thickness can increase to $8 \mathrm{~mm}$.
Most fascial ruptures and partial tears show an increase in thickness of the fascia of $10 \mathrm{~mm}$ or more with intrafascial high signal intensity of $\mathrm{T} 2$ weighted MR images.

In plantar fasciitis, the MR T2 weighted imaging or bright signal intensity is not actually seen within the fascia, but can be readily seen perifascially. If a bright signal is seen within the fascia, it will represent rupture of the fascial fibers confirming the diagnosis of plantar fascial rupture.

\section{References}

1. Theodorou, D.J., et al.: Plantar fasciitis and fascial rupture: MR imaging findings in 26 patients supplemented with anatomic data in cadavers. Radiographics. 20: S181- S197, 2000.

2. Lemont H, Ammirati, KM, Usen N: Plantar fasciitis: A degenerative process (fasciosis) without inflammation. $J \mathrm{Am}$ Podiatr Med Assoc 93(3): 234 - 237, 2003.

3. Barrett SL, O'Malley R.: Plantar fasciitis and other causes of heel pain. American Family Physician 59 (8), 1999.

4. Leach R, Jones R, Silva T: Rupture of the plantar fascia in athletes. J Bone Joint Surgery 60A (4): 537 - 539, 1978. 